\title{
Thromboelastographic Profile of Patients with Hyperparathyroidism Secondary to Chronic Kidney Failure Submitted to Total Parathyroidectomy -Case Series
}

\author{
Walkíria Wingester Vilas Boas, Cristiano Barbosa de Oliveira, Thadeu Alves Máximo, \\ Carlos Alexandre de Freitas Trindade, Alexandre de Andrade Sousa
}

Developed in Hospital das Clínicas da Universidade Federal de Minas Gerais HC-UFMG, Belo Horizonte, Brazil Email: walkiria589@hotmail.com

Received July $23^{\text {rd }}, 2013$; revised August $24^{\text {th }}, 2013$; accepted September $25^{\text {th }}, 2013$

Copyright (C) 2013 Walkíria Wingester Vilas Boas et al. This is an open access article distributed under the Creative Commons Attribution License, which permits unrestricted use, distribution, and reproduction in any medium, provided the original work is properly cited.

\begin{abstract}
Coagulopathy in surgical patients can cause perioperative complications, as both bleeding and thromboembolic events increase surgical morbimortality. The recognition of preexisting disorders and the understanding of the dynamic changes in hemostasis during surgery are prerequisites of safe patient management. The perioperative management of patients with chronic kidney failure is a huge challenge due to both the hypercoagulable state and increased risk of bleeding. Classic laboratory exams performed for the evaluation of blood clotting seem insufficient regarding the determination of the risk of bleeding and thrombosis in surgical patients. As patients with chronic kidney failure develop secondary hyperparathyroidism, the aim of the present study was to describe a case series and correlate the perioperative thromboelastographic profile of patients with chronic kidney failure submitted to parathyroidectomy with their secondary hyperparathyroidism.
\end{abstract}

Keywords: Thromboelastography; Chronic Renal Failure; Hyperparathyroidism; Total Thyroidectomy

\section{Background and Objectives}

Coagulopathy in surgical patients can cause perioperative complications, as both bleeding and thromboembolic events increase surgical morbimortality [1]. The recognition of preexisting disorders and the understanding of the dynamic changes in hemostasis during surgery are prerequisites of safe patient management.

While patients with chronic kidney failure are traditionally considered to be at risk for perioperative bleeding due to the platelet dysfunction related to uremia, anemia and frequent dialysis $[2,3]$, clotting disorders that favor a hypercoagulable state have also been demonstrated in such patients $[2,4,5]$. Therefore, the perioperative management of patients with chronic kidney failure is a huge challenge due to both the hypercoagulable state and increased risk of bleeding. This apparent dichotomy has important implications regarding clinical and anesthesiological management. A blood product reserve, pe- rioperative thromboprophylaxis and thrombosis prevention of the arteriovenous fistula are directly related to knowledge regarding the coagulation profile of patients with chronic kidney failure.

Classic laboratory exams performed for the evaluation of blood clotting seem insufficient regarding the determination of the risk of bleeding and thrombosis in surgical patients [6]. Thus, different dynamic coagulation monitors have been tested, including the thromboelastograph $[6,7]$. Thromboelastography allows the analysis of different phases of coagulation, the quality of the thrombus formed and fibrinolysis through the determination of five main indices on the readout. The $\mathrm{R}$ index (reaction time) is equivalent to the blood clotting time and has some correlation with other tests of the initial formation of fibrin. The $\mathrm{K}$ value (clot formation time) is the time the formed clot takes to reach a fixed degree of viscoelasticity. The alpha angle $(\alpha)$ is an indicator of the clot formation rate. Maximum amplitude (MA) is influenced 
by platelet function. The lysis index (Ly) is the determination of the lysis of the clot in time.

Thromboelastographic indices demonstrate that different coagulation aspects in the thromboelastographic readout are altered in patients with chronic kidney failure, including initial formation of fibrin (R), the fibrin-platelet interaction ( $\mathrm{K}$ and $\alpha$ ) and qualitative platelet function (MA) [2]. There is also a reduction in fibrinolysis [2,5]. The mechanisms of the increase blood clotting in patients with chronic kidney failure that lead to thrombotic complications are less known and seem have a multi-factor nature $[3,4]$. It has recently been demonstrated that patients with primary hyperparathyroidism and normal kidney function have a hypercoagulable and hypofibrinolytic profile, which may contribute to an increased risk of atherosclerotic and atherothrombotic complications [8].

As patients with chronic kidney failure develop secondary hyperparathyroidism, the aim of the present study was to describe a case series and correlate the perioperative thromboelastographic profile of patients with chronic kidney failure submitted to parathyroidectomy with their secondary hyperparathyroidism.

\section{Case Series}

The case series involved 11 patients with chronic kidney failure in dialysis treatment who were submitted to total parathyroidectomy with a forearm implant of a fragment of the parathyroid gland, with a mean surgical duration of two hours. All patients had hyperparathyroidism secondary to kidney failure and signed a statement of informed consent authorizing the collection of blood samples for perioperative laboratory exams and the publication of the findings. Regarding the etiology of chronic kidney disease, four cases were due to glomerulonephritis, two were due to polycystic kidneys, two were due to diabetic hypertensive nephropathy, one was due to nephrocalcinosis and two were due to hypertensive nephropathy. Table 1 displays the demographic data and results of the preoperative laboratory exams.

All patients were submitted to balanced general anesthesia (sufentanil $-0.5 \mu \mathrm{g} / \mathrm{kg}$; propofol $-2.5 \mathrm{mg} / \mathrm{kg}$; atracurium $-0.5 \mathrm{mg} / \mathrm{kg}$; sevorane-1.5 CAM) and monitored with continuous ECG, invasive blood pressure, pulse oximetry, gas analysis and capnography throughout the surgery. Arterial blood samples were collected immediately following anesthesia induction and at the end of the surgery (prior to extubation) for gasometry, ionogram, erythrogram, coagulogram and thromboelastography. The total intravenous volume administered during surgery was $462.5 \pm 50.42 \mathrm{~mL}$.

Table 2 displays the results of the laboratory exams and thromboelastogram of the patients in the intraopera- tive period.

The MA and $\alpha$ angle of the thromboelastogram immediately following anesthesia induction demonstrated a pro-thrombotic profile (means above values considered normal). In the comparison of laboratory exams after anesthesia induction and prior to extubation, only the number of platelets and ionic calcium underwent significant reductions, but remained within the range of reference values. A negative correlation was found between the parathyroid hormone (PTH) concentration and the K parameter of the thromboelastogram (Figure 1) and a positive correlation was found between PTH and the $\alpha$ angle of the thromboelastogram (Figure 2).

\section{Conclusions}

The thromboelastographic profile of the patients with chronic kidney failure and secondary hyperparathyroidism submitted to total parathyroidectomy with the forearm implant of parathyroid fragments demonstrated a state of prothrombotic coagulation. As in primary hyperparathyroidism [8], hyperparathyroidism secondary to

Table 1. Preoperative data.

\begin{tabular}{cc}
\hline Age & $44 \pm 3.9$ years \\
Gender & $8 / 3(\mathrm{M} / \mathrm{F})$ \\
Urea & $137.1 \pm 15.7 \mathrm{mg} / \mathrm{dl}$ \\
Creatinine & $8.7 \pm 0.9 \mathrm{mg} / \mathrm{dl}$ \\
Last preoperative dialysis & $22.5 \pm 0.6$ hours earlier \\
Parathyroid hormone $(\mathrm{n}=9)$ & $1753 \pm 170$ \\
\hline
\end{tabular}

Table 2. Comparative data before and just after anestehetic induction.

\begin{tabular}{ccc}
\hline & $\begin{array}{c}\text { After anesthesia } \\
\text { induction }\end{array}$ & $\begin{array}{c}\text { Before anethesia } \\
\text { induction }\end{array}$ \\
\hline Hemoglobine $(\mathrm{g} / \mathrm{dL})$ & $11.1 \pm 0.58$ & $10.67 \pm 0.48$ \\
Platelets $\left(/ \mathrm{mm}^{3}\right)$ & $207.875 \pm 19.837$ & $206.222 \pm 21.627^{*}$ \\
RNI & $1.13 \pm 0.02$ & $1.12 \pm 0.02$ \\
APTT $(\mathrm{p} / \mathrm{c})$ & $1.04 \pm 0.05$ & $0.99 \pm 0.05$ \\
Ionic Calcium $(\mathrm{mmol} / \mathrm{L})$ & $1.22 \pm 0.02$ & $1.13 \pm 0.02^{*}$ \\
R time & $26.23 \pm 1.62$ & $27.64 \pm 3.86$ \\
K time & $8.77 \pm 1.05$ & $9.29 \pm 1.29$ \\
Ang $\alpha$ & $47.07 \pm 3.70$ & $46.80 \pm 4.92$ \\
MA & $71.94 \pm 3.27$ & $61.28 \pm 3.40$ \\
Ly60 & 0 & 0 \\
\hline ângulo $\alpha$-VR $\left.=29^{\circ}-43^{\circ}\right)$ & &
\end{tabular}

${ }^{*} \mathrm{p}<0.05$ 


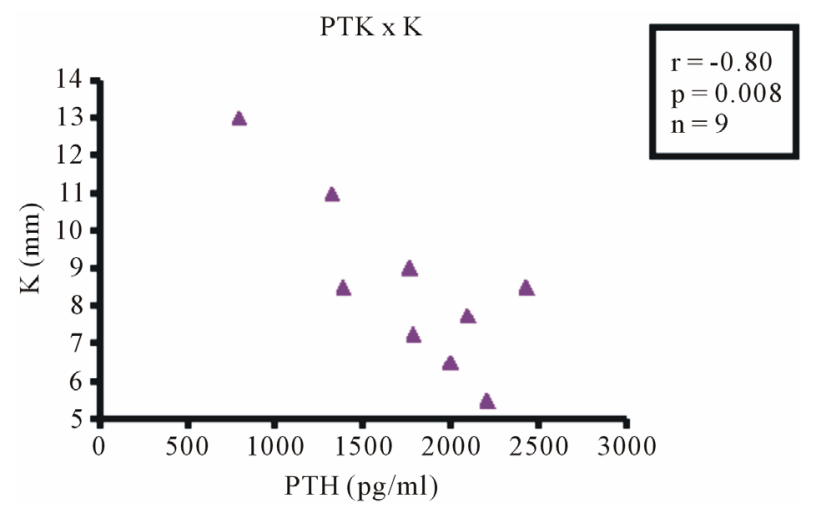

Figure 1. Negative correlation between parathormone and K thromboelastogram parameter.

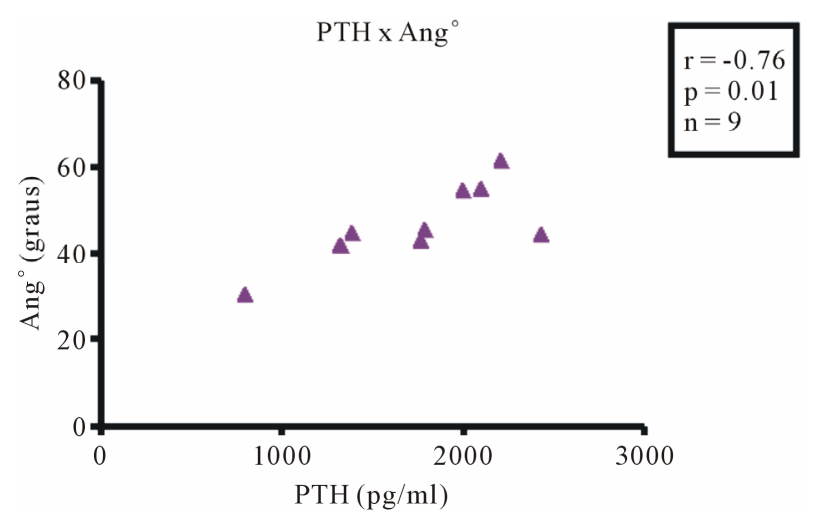

Figure 2. Positive correlation between parathormone and alpha angle thromboelastogram parameter.

chronic kidney failure may be related to the prothrombotic thromboelastographic profile presented.

While the traditional concern of anesthesiologists and surgeons during surgery on patients with chronic kidney failure is the possibility of greater surgical bleeding [9], clinical evidence has demonstrated that chronic kidney disease is also associated with blood clotting abnormalities that favor a pro-thrombotic hypercoagulable state $[3-5,10]$, with an increased risk of thromboembolic phenomena that may increase with the decline in kidney function [3]. While the risk of bleeding may be due to uremia (abnormal platelet function) and anemia (alteration from normal flow in blood vessels) [3,5,9], the tendency toward hypercoagulability likely also has a multifactor nature (increased levels of pro-coagulation factors, reductions in endogenous anticoagulants and fibrinolytic activity and the use of erythropoietin) [3]. The reduction in uremia with dialysis less than 24 hours prior to surgery helps improve platelet function [9]. The patients studied had a normal coagulogram, with no risk of bleeding. Indeed, no significant perioperative bleeding occurred in any of the surgeries. Dialysis on average $22.5 \pm 0.6$ hours prior to surgery may have contributed to adequate plate- let function despite the chronic kidney failure of the patients.

Important differences in hemostatic parameters have been demonstrated between patients with primary hyperparathyroidism (with normal kidney function) and healthy controls [8]. Such patients have a hypercoagulable and hypofibrinolytic profile [8]. The PTH levels of patients with primary hyperparathyroidism are correlated with this pro-thrombotic profile and likely contribute toward the increase in cardiovascular mortality [8]. The patients studied here had an indication for total parathyroidectomy due to hyperparathyroidism secondary to kidney failure. An increase in $\alpha$ angle and MA and reduction in fibrinolysis in 60 minutes were demonstrated in the patients studied, indicating a hypercoagulable pattern, as described in the literature [2]. The statistical analysis also demonstrated a positive correlation between PTH and tromboelastographic parameters (a higher PTH level denotes greater hypercoagulability). Therefore, as with primary hyperparathyroidism, secondary hyperparathyroidism in patients with chronic kidney failure may participate in the hypercoagulable state found in these patients.

The patients in the present study with chronic kidney failure and secondary hyperparathyroidism submitted to total parathyroidectomy exhibited pro-thrombotic tromboelastographic parameters. Hyperparathyroidism secondary to chronic kidney failure may be related to this hypercoagulable profile. If confirmed in larger clinical trials, the positive correlation between the pro-thrombotic state and PTH levels in patients with chronic kidney failure will likely lead to the development of strategies for the prophylaxis and treatment of thromboembolic events (including arteriovenous fistulas) and could be considered yet another criterion for parathyroidectomy in these patients.

\section{REFERENCES}

[1] P. Innerhofer and J. Kienast, "Principles of Perioperative Coagulopathy," Best Practice \& Research Clinical Anaesthesiology, Vol. 24, No. 1, 2010, pp. 1-14. http://dx.doi.org/10.1016/j.bpa.2009.09.006

[2] E. G. Pivalizza, D. C. Abramson and A. Harvey, "Perioperative Hypercoagulability in Uremic Patients: A Viscoelastic Study," Journal of Clinical Anesthesia, Vol. 9, No. 6, 1997, pp. 442-5. http://dx.doi.org/10.1016/S0952-8180(97)00097-4

[3] W. E. Dager and T. H. Kiser, "Systemic Anticoagulation Considerations in Chronic Kidney Disease," Advances in Chronic Kidney Disease, Vol. 17, No. 5, 2010, pp. 420427. http://dx.doi.org/10.1053/j.ackd.2010.06.002

[4] M. J. Adams, A. B. Irish and G. F. Watts, et al., "Hypercoagulability in Chronic Kidney Disease Is Associated 
with Coagulation Activation But Not Endothelial Function," Thrombosis Research, Vol. 123, No. 2, 2008, pp. 374-380.

http://dx.doi.org/10.1016/i.thromres.2008.03.024

[5] R. G. Craig and J. M. Hunter, "Recent Developments in the Perioperative Management of Adult Patients with Chronic Kidney Disease," British Journal of Anaesthesia, Vol. 101, No. 3, 2008, pp. 296-310. http://dx.doi.org/10.1093/bja/aen203

[6] D. Bischof, S. Dalbert, A. Zollinger, et al., "Thrombelastography in the Surgical Patient," Minerva Anestesiologica, Vol. 76, No. 2, 2010, pp. 131-137.

[7] J. L. Kashuk, E. E. Moore, A. Sabel, et al., "Rapid Thrombelastography (r-TEG) Identifies Hypercoagulability and Predicts Thromboembolic Events in Surgical Patients," Surgery, Vol. 146, No. 4, 2009, pp. 764-772. http://dx.doi.org/10.1016/j.surg.2009.06.054
[8] C. Erem, M. Kocak, I. Nuhoglu, et al., "Increased Plasminogen Activator Inhibitor-1, Decreased Tissue Factor Pathway Inhibitor, and Unchanged Thrombin-Activatable Fibrinolysis Inhibitor Levels in Patients with Primary Hyperparathyroidism," European Journal of Endocrinology, Vol. 160, No. 5, 2009, pp. 863-868. http://dx.doi.org/10.1530/EJE-09-0069

[9] D. Trainor, E. Borthwick and A. Ferguson, "Perioperative Management of the Hemodialysis Patient," Seminars in Dialysis, Vol. 24, No. 3, 2011, pp. 314-326. http://dx.doi.org/10.1111/j.1525-139X.2011.00856.x

[10] K. Wattanakit, M. Cushman, C. Stehman-Breen, et al., "Chronic Kidney Disease Increases Risk for Venous Thromboembolism," Journal of the American Society of Nephrology, Vol. 19, No. 1, 2008, pp. 135-140. http://dx.doi.org/10.1681/ASN.2007030308 